\title{
Individualismo y desconfianza en el trabajo asociativo de cadenas productivas de agroexportacion en el Valle del Mantaro, Junín, Perú
}

\author{
Individualism and distrust in the associative work of productive chains of \\ agro-export in the Mantaro Valley, Junín, Peru
}

William Rodríguez Giráldez

Universidad Continental

\section{RESUMEN}

Objetivos: Determinar el individualismo y desconfianza en el trabajo asociativo de cadenas productivas de agroexportación en el Valle del Mantaro. Métodos: Investigación descriptiva, no experimental, de alcance transeccional correlacional. Fueron encuestados 383 productores agrícolas de las provincias que comprenden el Valle del Mantaro: Huancayo, Concepción, Jauja y Chupaca. Se utilizaron los estadísticos descriptivos, distribución de frecuencias, tablas de contingencia, y chi cuadrado para prueba de hipótesis. Resultados: En la dimensión individualismo: $72,23 \%$ de productores manifestaron una actitud positiva hacia cadenas productivas; $87,2 \%$ estimaron importante participar en reuniones; 88,5\% consideraron importante la capacitación en asociatividad y 86,2 $\%$ gustan trabajar conjuntamente con vecinos. Se deduce que el $83,53 \%$ de ellos no tienen el rasgo de individualismo; por tanto, solo el 16,47 \% tienen este rasgo y no influye negativamente en el trabajo asociativo en el Valle del Mantaro. En la dimensión desconfianza: $52 \%$ de productores agrícolas manifestaron buena comunicación en la cadena productiva; $40 \%$ aportaron entre $50 \%$ y $100 \%$ de costos de producción; $56 \%$ podrían aportar entre $50 \%$ y $100 \%$ de costos de producción; $60 \%$ señalaron que confían en empresas articuladoras y $60 \%$ confían en colegas productores. Se deduce que, 53,6 \% de estos productores no poseen el rasgo de desconfianza; mientras que $46,4 \%$ si los tienen. Conclusiones: Solo 16,47 \% de productores agrícolas tienen rasgos de individualismo y no influye negativamente en el trabajo asociativo;

$46,4 \%$ de estos productores tienen el rasgo de desconfianza y este valor sí influye negativamente en el trabajo asociativo en el valle.

Palabras clave: Individualismo, desconfianza, trabajo asociativo, cadenas productivas, agroexportación. 


\section{ABSTRACT}

Objectives: To determine individualism and distrust in the partnership work of agroexport production chains in the Mantaro Valley. Methods: Descriptive research, nonexperimental, correlational transactional scope. 383 farmers from the Mantaro Valley provinces: Huancayo, Concepción, Jauja and Chupaca were surveyed. Descriptive statistics, frequency distribution, contingency tables and Chi square for hypothesis testing were used. Results: In the individualism dimension: $72,23 \%$ of farmers expressed a positive attitude to production chains; 87,2 $\%$ consider it's important to participate in meetings; $88,5 \%$ considered it's necessary the partnership training and $86,2 \%$ like to work together with neighbors. It follows that $83,53 \%$ of them don't have the individualism trait; therefore, only $16,47 \%$ have this trait and it's not adversely affecting the partnership work in the Mantaro Valley. In the mistrust dimension: $52 \%$ of farmers said that exists good communication in the production chain; $40 \%$ contribute between $50-100 \%$ of production costs; $56 \%$ could contribute between $50 \%$ and $100 \%$ of production costs; $60 \%$ rely on the articulating company and $60 \%$ rely on their farmers colleagues. It follows that $53,6 \%$ of these farmers don't have the mistrust trait; while $46,4 \%$ have it. Conclusions: Only $16,47 \%$ have the individualism trait and it's not adversely affecting the partnership work; $46,4 \%$ of these farmers have the mistrust trait and it's adversely affecting the partnership work in the valley.

Keywords: Individualism, distrust, partnership work, productive chains, agricultural exports.

\section{INTRODUCCIÓN}

Durante muchos años se han formado diferentes cadenas productivas de agroexportación en el Valle del Mantaro, Junín, y en las regiones vecinas, financiadas por cooperación técnica internacional, programas del Estado y otros; sin embargo, al pasar el tiempo, y terminar el proyecto, estas se ha disuelto y son muy pocas las que continúan trabajando.

Una experiencia destacable en la región central es lo ocurrido con el proyecto de la cadena productiva de tuna y cochinilla en Ayacucho, proyecto ejecutado por el Instituto de Investigación para el Desarrollo Agroindustrial (IIPDA), y financiado por el convenio ADEX-AID/ MSP (1). Desde su creación (1997-1999), el IIPDA ha organizado 45 comités de productores de tuna y cochinilla, y 20 comités de tuna, donde se han prestado servicios de capacitación y asistencia técnica a cerca de 1100 pequeños productores, con el fin de elevar la producción, productividad, calidad y generar mayores ingresos y empleos de los recolectores de tuna y cochinilla en el departamento de Ayacucho y Huancavelica. Del total de las organizaciones promovidas por esta institución, permanecen aún operativos el $5 \%$ y, de ellos, la mayoría se han convertido en organizaciones familiares, solo para la comercialización de tuna, fruta principalmente de Huanta y Pacaycasa.

Otra experiencia es IDESI Ayacucho, desde 1998 hasta el 2008, con el financiamiento de las instituciones: DOEN, FONDOEMPLEO, FONCODES, INCAGRO y Proyecto IPYMER, promovió más de 30 organizaciones de productores de tuna y cochinilla, en Ayacucho y Huancavelica, con los componentes de organización, capacitación, asistencia técnica, micro créditos y articulación comercial, de las cuales sólo ocho están operativas.

La reducida cantidad de organizaciones productivas que quedan operativas tras su auspiciosa creación, es la consecuencia de la dificultad que tienen las diversas organizaciones de la región central y del propio Valle del Mantaro para emprender de manera integrada, asociada. Benavides (2) señala que siempre está latente el tema de la desintegración. Todas estas asociaciones todavía son débiles institucionalmente. Antes, Prompex les pagaba al articulador y ahora que ya no cuentan con ese apoyo y todas las empresas deben aportar algo 
económicamente, empiezan a surgir las divisiones.

Según el estudio sobre Cluster y Asociatividad, el logro del cluster quesero de Bambamarca en la zona central de Cajamarca (3) fue el afán de formación de una asociación de pequeños productores que buscaba detener la confusión de su producto con otros que son de menor calidad. Por ello, se buscó emprender una lucha contra la piratería de la denominación de origen del queso mantecoso y tipo suizo de Cajamarca. Sin embargo, a pesar de los esfuerzos privados por generar una sólida asociación entre los productores así como la obtención de una marca colectiva, no fue suficiente para una acción efectiva pues aún muchos actores no olvidan sus estrategias "individuales". Existe desconfianza en la asociación con productores paralelos, lo que revela un gran problema de capital social en la zona.

Otra carencia es la inexistencia de una red de comunicación entre los productores artesanos y comerciantes. Boucher (4) arguye que en dicho cluster existe un círculo vicioso de engaños consentidos entre los agentes del cluster. Es decir, todos prometen realizar una mejora pero ninguno la realiza, en espera que sea el otro agente quien inicie esta acción. $Y$ sin lugar a dudas, el mayor problema de Bambamarca es la fuente de competencia de vendedores ilegales y producción alterada o de mala calidad que se vende como producto de la zona. La cooperación ha sido una estrategia auspiciada por los organismos que trabajan en la zona pero aún prevalece la desconfianza entre los productores paralelos, por lo cual, dicha estrategia no se consolida del todo.

El Perú es un país que sufre de falta de confianza interpersonal. La confianza es un factor de articulación, de asociación y de motivación para la acción conjunta y por lo tanto es un elemento clave y determinante para el afianzamiento de los esquemas asociativos de organización empresarial, tanto más para el fortalecimiento de los clusters. El Latinobarómetro (5) del 2002 muestra los resultados de la pregunta de si confía en la mayoría de las personas o cree que uno nunca es lo suficientemente cuidadoso en el trato con los demás. El Perú ocupa en el sondeo el undécimo lugar de un total de diecisiete países con 15 puntos, muy por debajo del promedio necesario de 18522 .

Estos resultados denotan que para fomentar las distintas formas de asociatividad y el robustecimiento de los clusters en el país, es imprescindible revertir el problema de la falta de confianza. Como se sabe, no existe un estudio exhaustivo de las razones de la desconfianza y se debería realizar esfuerzos para construir un modelo de decisión de manera que se puedan identificar los incentivos que permitan revertir este problema.

En el Perú, según Castillo (6), es muy difícil trabajar bajo esquemas asociativos por el interés individualista que tienen los empresarios. La interacción es el elemento más difícil. Los gremios juegan un rol importante para hacer que se conozcan, se capaciten y tomen la decisión de trabajar conjuntamente.

Arteaga (7), señala que los resultados del Censo Agropecuario 2012 (8) dieron como resultado que actualmente, hay medio millón de agricultores más que hace 18 años. El agricultor, dueño de una hectárea, tiene hijos y nietos, quienes heredaron estas tierras. El problema de esta realidad es que resta competitividad, y si a eso se suma que el $73 \%$ de los agricultores tiene menos de tres hectáreas y el $90 \%$ menos de diez, esto se agrava. El desafío está en hacer que estos agricultores se inserten en la economía dinamizada, en la que ya se encuentra el país.

En agroexportaciones el Perú ha pasado de 300 millones de dólares de exportación en 1990 a 4700 millones en el 2012. La solución para estos pequeños agricultores está en la asociatividad, pero ésta debe ser eficiente y se logra a través de tres pilares: El primero es la gestión, el líder de una asociación debe tener visión empresarial y 
global, capacidad técnica y empatía con los agricultores. El segundo es el financiamiento donde existen ya ciertas ofertas en el mercado dirigidas a este público. El tercero, está el acompañamiento y seguimiento.

El $45 \%$ de las empresas creadas en el Perú desaparece en el primer año; el $75 \%$ en el cuarto y la realidad de las asociaciones agropecuarias no va a ser diferente. La asociación es la fórmula, pero ésta no debe ser impuesta sino voluntaria. Se debe crear incentivos para que los agricultores se integren y hagan asociaciones en conjunto.

En productividad el Perú no es competitivo en cultivos estratégicos nacionales donde invierte la gran mayoría de los agricultores. El rendimiento promedio nacional en toneladas por hectárea en maíz en el Perú es de 3, mientras que en Chile y Estados Unidos es de 10. En el caso de la papa es 13 toneladas por hectárea en el Perú, 20 en Chile, 24 en Brasil, 46 en Estados Unidos y 47 en México. Además, la agricultura en el Perú tiene diferentes matices; por un lado, hay empresas de primer nivel con cientos de hectáreas, y algunas de ellas con miles de hectáreas cada una, con diversificación de productos, tecnología de punta y produciendo en más de una región; pero el $95 \%$ de los predios agrarios en el Perú tienen menos de 20 hectáreas, y el $81 \%$ tienen menos de 5 hectáreas (7).

Scott, en el estudio Competitividad agrícola y desarrollo de cadenas o clústeres de valor en el Perú (9), señala que durante 2001 2009 el sector agrícola creció a una tasa promedio anual de 4,2 \% y la Balanza Comercial Agraria pasó de negativa a positiva en 2004. No obstante, sólo pocos productos (producción agropecuaria, agroexportaciones), están concentrados tanto en producción física en toneladas métricas (TM) como en el valor del mismo. De todas maneras, dado que la economía se encuentra en un proceso de profundización y extensión de su apertura comercial, que implica la suscripción de acuerdos comerciales con otros países, lo que ha sido notable en los últimos años ha sido la tendencia para producir y vender toda una gama de nuevos productos tanto al extranjero como al mercado nacional.

En el Valle del Mantaro se han realizado esfuerzos de trabajos asociativos, en cadenas productivas promovidas por entidades públicas y privadas como ONG o sociedades civiles sin fines de lucro y se ofrecieron servicios de articulación al mercado, asociatividad con empresas agroexportadoras, capacitación y asistencia técnica y financiamiento de la producción, etc.; sin embargo, no se han logrado los resultados esperados y las cadenas productivas ya no operan o ya no existen. Entonces se plantean interrogantes como: ¿̇qué ha sucedido con las cadenas productivas en el Valle del Mantaro?, ¿̇por qué no se tienen estadísticas de cadenas productivas que permanecen en el mercado?, ¿̇qué otras razones diferentes a las técnicas, de mercado y financieras, influyen en el sostenimiento y desarrollo de las cadenas productivas?, ¿̇cómo se manifiestan los factores culturales como el individualismo y la desconfianza, entre otros factores, en los productores del Valle del Mantaro?, ¿̇cómo influyen estos factores culturales en el trabajo asociativo de las cadenas productivas?, etc.

En los estudios realizados sobre asociatividad y clusters, si bien se les nombra, no se han identificado y profundizado los temas relacionados a los factores culturales o variables "suaves", no medibles, como la confianza, compromiso social, cooperación, competencia, etc., que influyen en el sostenimiento y desarrollo de cadenas productivas en los contextos determinados. En ese sentido, se planteó el siguiente problema de investigación: ¿̇En qué medida el individualismo y la desconfianza influyen en el trabajo asociativo de las cadenas productivas de agroexportación en el Valle del Mantaro, Junín?, con el objetivo de determinar cómo el individualismo y la desconfianza influyen en el trabajo asociativo de las cadenas productivas de agroexportación en el Valle del Mantaro, Junín.

Sobre la base teórica sobre asociatividad y 
cultura se plantearon las siguientes hipótesis: 1) El individualismo influye negativamente en el trabajo asociativo de las cadenas productivas de agroexportación en el Valle del Mantaro, Junín y 2) la desconfianza influye negativamente en el trabajo asociativo de las cadenas productivas de agroexportación en el Valle del Mantaro, Junín.

Los factores culturales tienen importancia en el trabajo asociativo y en el desarrollo de cadenas productivas en el Valle del Mantaro. A pesar de contar con apoyo tecnológico, créditos o aportes de la empresa a los costos de producción, capacitación y asistencia técnica, acceso a mercados, apoyo del Estado a través de la DGPA del Ministerio de Agricultura, alguna ONG o empresa articuladora, no se han encontrado cadenas productivas que permanezcan en el mercado con indicadores exitosos, sin considerar aspectos de la cultura o creencias de los productores. En el Valle del Mantaro no se conoce aún una empresa asociativa exitosa y en crecimiento, en todo caso se cuenta con poca información estadística de las cadenas productivas en operaciones. El estudio solo considera dos factores culturales: la individualidad y la desconfianza y su influencia en el trabajo asociativo de cadenas productivas; sin embargo, hay otros factores como la informalidad, la toma de decisiones, la escasa planificación de la producción, etc., que podrán ser parte de otros trabajos de investigación.

Los resultados del trabajo de investigación servirán como base para la formación y desarrollo de nuevas cadenas productivas en el Valle del Mantaro. Conocer la influencia de factores culturales como el individualismo y la desconfianza en la formación, permanencia, crecimiento y logro de rentabilidad de la cadena productiva en el mercado nacional e internacional, traerá como consecuencia la mejora de la calidad de vida de los productores y para muchos salir de la pobreza y extrema pobreza. Posteriormente los resultados del estudio servirán también para aplicarlos en otros valles del Perú, principalmente de la sierra, por sus características particulares.

\section{MATERIAL Y MÉTODOS}

El nivel de investigación fue descriptivo, no experimental, transeccional o transversal de alcance correlacional (10). La población considerada en el estudio es la población rural del Valle del Mantaro, según el INEI (11) es de 132459 habitantes, repartidos por provincias: Huancayo (41 \%), Concepción (22\%), Jauja (21\%) y Chupaca (16\%). La muestra fue de 383 productores del Valle del Mantaro, con un margen de error permitido de $5 \%$, un factor $P$ de 0,5 y $Q$ de 0,5 , que son los máximos valores para esta proporción. Se aplicó un muestreo probabilístico aleatorio estratificado por el número de habitantes del sector rural de cada provincia del Valle del Mantaro.

La técnica de recolección de datos fue la encuesta y el instrumento fue el cuestionario (10), con preguntas cerradas de tipo nominal ya que se trató de encontrar la percepción de los productores de cada factor cultural que sentía en relación al trabajo asociativo en cadenas productivas. El procesamiento de los datos se realizó utilizando software especializado, estadísticas como distribución de frecuencias y tablas de contingencias o tablas cruzadas. Para probar las hipótesis, por la naturaleza de las variables de tipo nominal, fueron realizadas pruebas de chi cuadrado para determinar la relación entre las variables.

La variable individualismo se trabajó con las siguientes dimensiones: actitud negativa hacia cadenas productivas, el no querer participar en reuniones de la cadena, no dar importancia a la capacitación en asociatividad y no dar importancia al trabajo conjunto o acción conjunta con vecinos. La variable desconfianza se trabajó con las siguientes dimensiones: comunicación, cuanto aportó en una cadena productiva anterior, cuánto estaría dispuesto a aportar en una nueva cadena productiva, grado de confianza en empresa articuladora y confianza en colegas productores. 


\section{RESULTADOS}

Los resultados del trabajo de investigación permiten conocer la existencia de rasgos de individualismo y desconfianza en los productores, para el trabajo asociativo de la cadena productiva de agroexportación en el Valle del Mantaro.

\section{Resultados de la dimensión individualismo}

Los productores que tienen actitud muy positiva y positiva se encuentran en las provincias de Huancayo (38\%) y Concepción (18\%), (tabla $N^{\circ} 1$ ), que serán las indicadas para formar una nueva cadena productiva. En todo el Valle hay un total de $74,72 \%$ de actitud muy positiva y positiva hacia cadenas productivas, lo que indica que hay buena predisposición a trabajar en asociatividad.

La tabla $N^{\circ} 2$ muestra que el 10,68\% de los productores tiene una actitud muy positiva y $61,57 \%$ una actitud positiva hacia las cadenas productivas; por tanto el 72,25\% de productores tienen una actitud positiva a trabajar de manera asociativa en una cadena productiva. En contraste, solo el $2,13 \%$ de los productores tienen una actitud negativa hacia las cadenas productivas y $23,13 \%$ una actitud regular. La prueba de chi cuadrado $(X 2=13,695)$ muestra que hay relación entre las dos variables.

El $87,2 \%$ de productores sí acepta participar de reuniones relacionadas al trabajo asociativo de cadenas productivas (tabla $N^{\circ} 3$ ). En contraste el $9,9 \%$ no participa definitivamente de reuniones y los que si participan de reuniones pero no desean trabajar en una cadena productiva son el 2,9\%. La prueba de chi cuadrado $(X 2=121,227)$ indica que si existe relación entre las variables trabajo asociativo en cadena productiva y participación en reuniones.

En cuanto a la importancia de la capacitación en asociatividad, la tabla $\mathrm{N}^{\circ}$ 4 muestra que el $27,3 \%$ de productores consideran muy importante y $61,2 \%$ importante la capacitación en trabajo en equipo. Solamente el 0,3\% de productores consideran no importante y $7 \%$ más o menos importante. Además solo el 4,2\% $(0,7 \%+3,5 \%)$ si consideran importante la capacitación pero no desean participar en una cadena productiva. La prueba de chi cuadrado $(X 2=20,518)$ indica que existe relación entre las variables trabajo

Tabla $N^{\circ}$ 1: Actitud de los productores del Valle del Mantaro por provincias hacia cadenas productivas.

\begin{tabular}{llccccc}
\hline & & \multicolumn{4}{c}{ Provincia } & \multirow{2}{*}{ Total } \\
\cline { 3 - 5 } & & Huancayo & Jauja & Concepción & Chupaca & \\
\hline \multirow{2}{*}{ Actitud a } & Muy positiva & $5 \%$ & $1 \%$ & $4 \%$ & $1 \%$ & $11 \%$ \\
cadenas & Positiva & $33 \%$ & $6 \%$ & $14 \%$ & $12 \%$ & $65 \%$ \\
productivas & Regular & $11 \%$ & $6 \%$ & $5 \%$ & $1 \%$ & $23 \%$ \\
& Negativa & $1 \%$ & $0 \%$ & $0 \%$ & $0 \%$ & $1 \%$ \\
\hline & Total & $50 \%$ & $13 \%$ & $23 \%$ & $14 \%$ & $100 \%$ \\
\hline
\end{tabular}

Tabla $N^{\circ}$ 2: Actitud hacia cadenas productivas y trabajo asociativo en cadena agroexportadora.

\begin{tabular}{|c|c|c|c|c|c|c|c|}
\hline & & \multicolumn{5}{|c|}{ Actitud hacia cadenas productivas } & \multirow{2}{*}{ Total } \\
\hline & & $\begin{array}{c}\text { Muy } \\
\text { positiva }\end{array}$ & Positiva & Regular & Negativa & $\begin{array}{c}\text { Muy } \\
\text { negativa }\end{array}$ & \\
\hline Trabajo asociativo en & $\mathrm{Si}$ & $10,68 \%$ & $61,57 \%$ & $20,99 \%$ & $1,42 \%$ & $0,0 \%$ & $94,66 \%$ \\
\hline cadena productiva & No & $0,0 \%$ & $2,49 \%$ & $2,14 \%$ & $0,71 \%$ & $0,0 \%$ & $5,34 \%$ \\
\hline Total & & $10,68 \%$ & $64,06 \%$ & $23,13 \%$ & $2,13 \%$ & $0,0 \%$ & $100 \%$ \\
\hline
\end{tabular}


Tabla N ${ }^{\circ}$ 3: Participación en reuniones y trabajo asociativo.

\begin{tabular}{|c|c|c|c|c|}
\hline & \multicolumn{3}{|c|}{ Participa en reuniones } & \multirow{2}{*}{ Total } \\
\hline & & $\mathrm{Si}$ & No & \\
\hline Trabajo asociativo en & $\mathrm{Si}$ & $87,2 \%$ & $4,4 \%$ & $91,6 \%$ \\
\hline Cadena productiva & No & $2,9 \%$ & $5,5 \%$ & $8,4 \%$ \\
\hline Total & & $90,1 \%$ & $9,9 \%$ & $100 \%$ \\
\hline
\end{tabular}

Tabla $N^{\circ}$ 4: Importancia de capacitación en asociatividad y trabajo asociativo en cadena productiva.

\begin{tabular}{|c|c|c|c|c|c|c|}
\hline & & \multicolumn{4}{|c|}{ Capacitación } & \multirow{2}{*}{ Total } \\
\hline & & $\begin{array}{c}\text { Muy } \\
\text { importantes }\end{array}$ & Importantes & $\begin{array}{c}\text { Más o menos } \\
\text { importantes }\end{array}$ & $\begin{array}{c}\text { No } \\
\text { importantes }\end{array}$ & \\
\hline Trabajo asociativo en & $\mathrm{Si}$ & $27,3 \%$ & $61,2 \%$ & $6,6 \%$ & $0,0 \%$ & $95,1 \%$ \\
\hline Cadena productiva & No & $0,7 \%$ & $3,5 \%$ & $0,3 \%$ & $0,3 \%$ & $4,9 \%$ \\
\hline Total & & $28,0 \%$ & $64,7 \%$ & $7,0 \%$ & $0,3 \%$ & $100 \%$ \\
\hline
\end{tabular}

Tabla N ${ }^{\circ}$ 5: Trabajo conjunto con vecinos y trabajo asociativo en cadena productiva.

\begin{tabular}{|c|c|c|c|c|}
\hline & & \multicolumn{2}{|c|}{ Trabajo conjunto con vecinos } & \multirow{2}{*}{ Total } \\
\hline & & Si & No & \\
\hline Trabajo asociativo en & $\mathrm{Si}$ & $86,2 \%$ & $5,5 \%$ & $91,7 \%$ \\
\hline cadena productiva & No & $3,9 \%$ & $4,4 \%$ & $8,3 \%$ \\
\hline Total & & $90,1 \%$ & $9,9 \%$ & $100 \%$ \\
\hline
\end{tabular}

Tabla N ${ }^{\circ}$ 6: Grado de comunicación y trabajo asociativo en cadena productiva.

\begin{tabular}{|c|c|c|c|c|c|c|c|}
\hline & \multicolumn{7}{|c|}{ Grado de Comunicación } \\
\hline & & $\begin{array}{l}\text { Totalmente } \\
\text { comunicada }\end{array}$ & Comunicada & $\begin{array}{l}\text { Más o menos } \\
\text { comunicada }\end{array}$ & $\begin{array}{c}\text { No } \\
\text { comunicada }\end{array}$ & $\begin{array}{c}\text { Totalmente } \\
\text { nada } \\
\text { comunicada }\end{array}$ & Total \\
\hline Trabajo asociativo en & Si & $3,8 \%$ & $47,8 \%$ & $38,7 \%$ & $3,8 \%$ & $0 \%$ & $94,1 \%$ \\
\hline cadena productiva & No & $0 \%$ & $1,1 \%$ & $4,3 \%$ & $0 \%$ & $0,5 \%$ & $5,9 \%$ \\
\hline Total & & $3,8 \%$ & $48,9 \%$ & $43 \%$ & $3,8 \%$ & $0,5 \%$ & $100 \%$ \\
\hline
\end{tabular}

Tabla N ${ }^{\circ}$ 7: Porcentaje que aportó para cubrir costos de producción y trabajo asociativo en cadena productiva.

\begin{tabular}{lcccccccccc}
\hline & \multicolumn{10}{c}{ Qué \% aportó en cadena productiva } \\
& & $0 \%$ & $5 \%$ & $10 \%$ & $20 \%$ & $50 \%$ & $70 \%$ & $100 \%$ & Total \\
\hline Trabajo asociativo en & Si & $2,2 \%$ & $3,8 \%$ & $11,4 \%$ & $36,96 \%$ & $20,1 \%$ & $16,3 \%$ & $3,8 \%$ & $94,6 \%$ \\
cadena productiva & No & $1,1 \%$ & $1,1 \%$ & $0 \%$ & $2,17 \%$ & $1,1 \%$ & $0 \%$ & $0 \%$ & $5,4 \%$ \\
Total & & $3,3 \%$ & $4,9 \%$ & $11,4 \%$ & $39,13 \%$ & $21,2 \%$ & $16,3 \%$ & $3,8 \%$ & $100 \%$ \\
\hline
\end{tabular}


asociativo en cadenas productivas $y$ capacitación.

La tabla $N^{\circ} 5$ muestra que el 86,2 \% de productores consideran que sí es importante trabajar en conjunto con vecinos $y$ en contraste solo el 9,9\% consideran que no. Asimismo el 3,9\% consideran que sí es importante trabajar en conjunto con vecinos, pero no desean participar en una cadena productiva. La prueba de chi cuadrado $(X 2=72,924)$ indica que hay relación entre estas variables.

\section{Resultados de la dimensión desconfianza}

La tabla $N^{\circ} 6$ señala que el $0,5 \%$ de los productores manifiesta que la cadena productiva estuvo totalmente nada comunicada, 3,8 \% no comunicada y 43 $\%$ más o menos comunicada, haciendo un $47,3 \%$ la percepción de no comunicada. En contraste el estudio muestra que el 47,8 $\%$ de los productores manifiestan que hubo comunicación entre la empresa articuladora y los productores y 3,8\% totalmente comunicada. La prueba de chi cuadrado $(X 2=21,448)$ señala que hay relación entre las variables trabajo asociativo en cadena productiva y grado de comunicación.

La tabla $\mathrm{N}^{\circ} 7$ muestra los resultados de cuánto aportó el productor en una cadena productiva. El 58,74\% de los productores aportó de $0 \%$ a $20 \%$ de los costos de producción. Este dato señala que más de la mitad de productores solo aportó de 0 $\%$ a $20 \%$ de los costos de producción, lo que indica que no están involucrados con el negocio y no están dispuestos a apostar por el proyecto de cadena productiva. El 39,13 $\%$ de los productores aportó solo el $20 \%$; un 11,4 \% aportó sólo el $10 \%$; un 4,9\% aportó solo el 5 y un 3,3 \% aportó $0 \%$.

En contraste, un 20,1 \% de productores aportó el $50 \%$ de los costos de producción, un $16,3 \%$ aportó un $70 \%$ y un 3,8 \% aportó el $100 \%$ de los costos de producción. En resumen, un 40,2 \% de productores aportó entre $50 \%$ y $100 \%$ de los costos de producción. El hecho de aportar más para los costos de producción señala que los productores están dispuestos a apostar por el negocio y tienen mayor actitud de involucramiento y confianza en el proyecto de la cadena productiva. La prueba de chi cuadrado $(X 2=17,364)$ señala que hay relación entre las variables trabajo asociativo en cadena productiva y aporte de costos de producción.

En relación con el porcentaje de cuánto están dispuestos a aportar en una nueva cadena productiva de agroexportación, la tabla $N^{\circ} 8$ indica que el $41 \%$ de los productores aportaría de $0 \%$ a $25 \%$ de los costos de producción (27\% aportaría $25 \%$ de los costos, $14 \%$ solo el $10 \%$ y $1 \%$ no aportaría nada). En contraste, un $56 \%$ de productores están dispuestos a aportar entre un $50 \%$ y $100 \%$ de los costos de producción. Un $33 \%$ aportaría el $50 \%$ de los costos, un $13 \%$ el $75 \%$ y un $10 \%$ aportaría el $100 \%$. La prueba de chi cuadrado $(X 2=21,122)$ señala que hay relación entre estas variables.

La tabla $\mathrm{N}^{\circ} 9$ indica que el $8 \%$ de productores desconfían de una empresa agroexportadora y un $30 \%$ más o menos confían. En contraste un $54 \%$ si confían y un $6 \%$ confían totalmente en una empresa articuladora, pero que ofrezcan garantía, responsabilidad, mercado seguro y propuestas claras. La prueba de chi-cuadrado $(X 2=55,065)$ señala que hay relación entre las variables trabajo asociativo en cadena productiva y confianza en empresa articuladora.

Además, se les preguntó a los productores, ¿qué aspectos solicitarían a la empresa articuladora para confiar en ella y formar una cadena productiva?. Los resultados fueron: Un 46,1 \% de productores exigen garantía y responsabilidad; un 29,8\% mercado seguro o que los precios de los productos se mantengan; un 12,2\% propuestas claras sin cambios posteriores en relación al precio o condiciones técnicas o de calidad, devolución de productos, un $8,9 \%$ exige apoyo tecnológico y otras con porcentajes más pequeños, como se puede apreciar en la figura $N^{\circ} 1$. 
Tabla $N^{\circ}$ 8: Porcentaje dispuesto a aportar para costos de producción y trabajo asociativo en cadena productiva.

\begin{tabular}{|c|c|c|c|c|c|c|c|c|}
\hline & & \multicolumn{6}{|c|}{ Qué \% aportaría en cadena productiva } & \multirow{2}{*}{ Total } \\
\hline & & $0 \%$ & $10 \%$ & $25 \%$ & $50 \%$ & $75 \%$ & $100 \%$ & \\
\hline Trabajo asociativo en & $\mathrm{Si}$ & $1 \%$ & $11 \%$ & $24 \%$ & $33 \%$ & $13 \%$ & $10 \%$ & $92 \%$ \\
\hline cadena productiva & No & $0 \%$ & $3 \%$ & $3 \%$ & $1 \%$ & $1 \%$ & $0 \%$ & $8 \%$ \\
\hline Total & & $1 \%$ & $14 \%$ & $27 \%$ & $34 \%$ & $14 \%$ & $10 \%$ & $100 \%$ \\
\hline
\end{tabular}

Tabla $N^{\circ}$ 9: Confianza en empresa articuladora y trabajo asociativo en cadena productiva.

\begin{tabular}{|c|c|c|c|c|c|c|c|}
\hline & & \multicolumn{5}{|c|}{ Confía en empresa articuladora } & \multirow[b]{2}{*}{ Total } \\
\hline & & $\begin{array}{c}\text { Confío } \\
\text { totalmente }\end{array}$ & Si confío & $\begin{array}{l}\text { Más o menos } \\
\text { confío }\end{array}$ & Desconfío & $\begin{array}{l}\text { Desconfío } \\
\text { totalmente }\end{array}$ & \\
\hline Trabajo asociativo en & $\mathrm{Si}$ & $6 \%$ & $54 \%$ & $27 \%$ & $5 \%$ & $0 \%$ & $92 \%$ \\
\hline Cadena productiva & No & $0 \%$ & $2 \%$ & $3 \%$ & $3 \%$ & $0 \%$ & $8 \%$ \\
\hline Total & & $6 \%$ & $56 \%$ & $30 \%$ & $8 \%$ & $0 \%$ & $100 \%$ \\
\hline
\end{tabular}

Tabla $N^{\circ}$ 10: Confianza en colegas productores y trabajo asociativo en cadena productiva.

\begin{tabular}{lcccccc}
\hline & \multicolumn{1}{c}{ Confía en colegas productores } & & & \\
\hline & $\begin{array}{c}\text { Confío } \\
\text { totalmente }\end{array}$ & Si confio & $\begin{array}{c}\text { Más o menos } \\
\text { confio }\end{array}$ & Desconfio & Total \\
\hline Trabajo asociativo en & $\mathrm{Si}$ & $4,7 \%$ & $55 \%$ & $28,8 \%$ & $3,1 \%$ & $91,6 \%$ \\
cadena productiva & $\mathrm{No}$ & $0 \%$ & $2,1 \%$ & $4,7 \%$ & $1,6 \%$ & $8,4 \%$ \\
Total & $4,7 \%$ & $57,1 \%$ & $33,5 \%$ & $4,7 \%$ & $100 \%$ \\
\hline
\end{tabular}

Para que una empresa articuladora forme una cadena agroexportadora tendrá que tener en cuenta que es importante para los productores la garantía y responsabilidad, el mercado seguro y tener propuestas claras. Es decir garantía y responsabilidad para compartir los riesgos del negocio, tener bien en claro el mercado a donde se va a atender, tener bajo control los precios a los cuales se va a negociar y tener los estudios técnicos que permitan obtener la productividad adecuada para tener costos calculados que permitan obtener utilidades, estudios de mercado que permitan generar propuestas claras de comercialización y obtener los resultados de rentabilidad del negocio propuesto para la cadena productiva de agroexportación.

En relación con la confianza hacia los colegas productores, la tabla $\mathrm{N}^{\circ} 10$ indica, que un $4,7 \%$ desconfía en sus colegas productores y un $33,5 \%$ más o menos confía. En contraste un $55 \%$ si confía y un $4,7 \%$ confían totalmente en sus colegas productores, para formar una nueva cadena agroexportadora, indicándonos que la desconfianza en sus colegas productores no se ha manifestado en un $59,7 \%$ de los productores del Valle del Mantaro. La prueba de chi-cuadrado señala que hay relación entre estas variables.

\section{DISCUSIÓN}

En la dimensión individualismo, si se suma las respuestas muy positivas y positivas como elementos contrarios al del rasgo individualismo se tiene que el $72,25 \%$ 


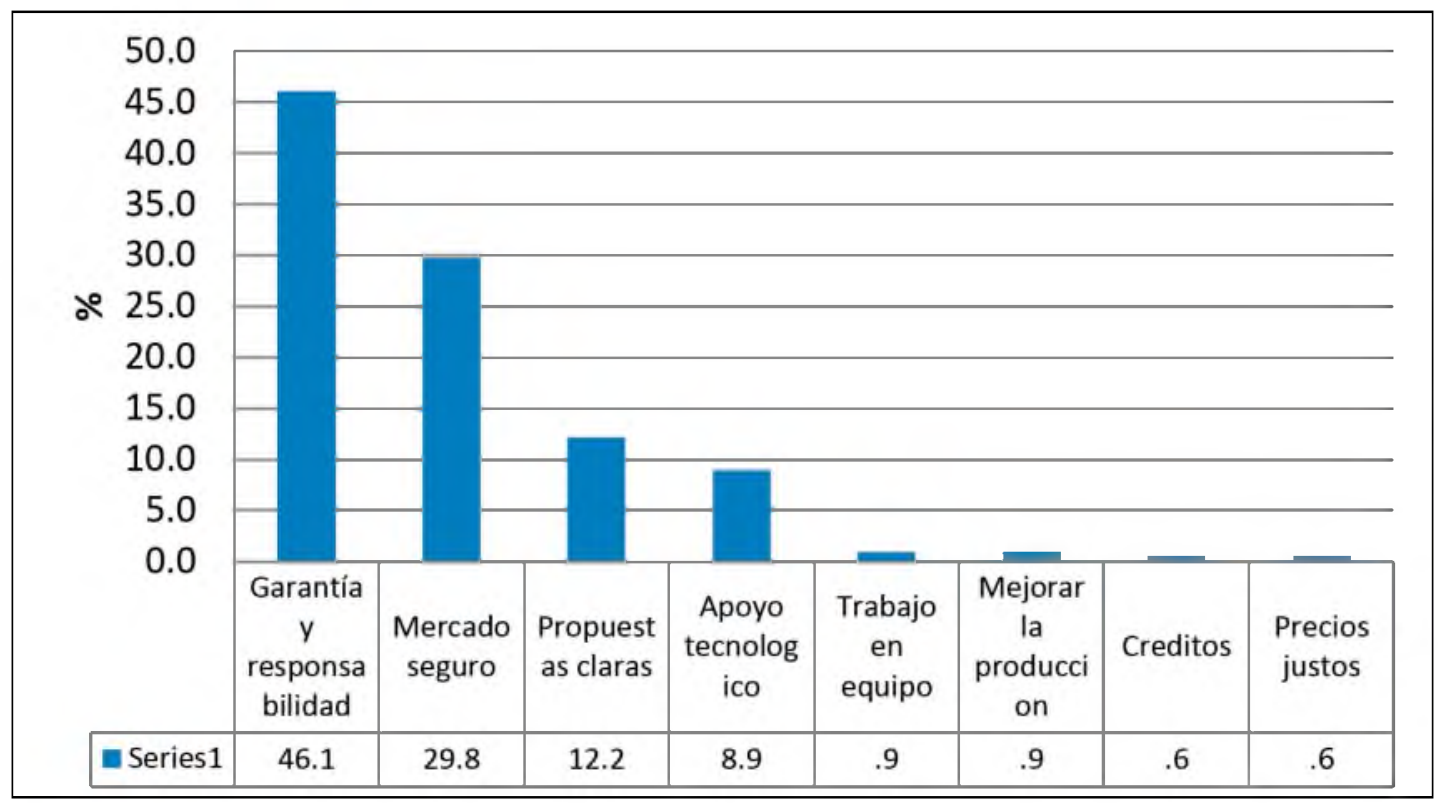

Figura $N^{0} 1$ : Aspectos que solicitan los productores a la empresa articuladora para formar una cadena productiva de agroexportación.

de productores tienen actitud positiva a la cadenas productivas; $87,2 \%$ sí tienen actitud positiva para participar de las reuniones de la cadena productiva; 88,5 $\%$ consideran importante la capacitación en temas productivos, asociatividad, trabajo en equipo y 86,2 \% si gustan trabajar conjuntamente con vecinos. Se deduce entonces que por lo menos el $83,53 \%$ de los productores agrícolas del Valle del Mantaro no tienen el rasgo de individualismo; por tanto, en relación con la hipótesis 1, se deduce que el rasgo de individualismo solo se presenta en el 16,47 $\%$ de los productores del Valle del Mantaro y esta no influye negativamente en el trabajo asociativo de las cadenas productivas en el Valle del Mantaro, Junín, entonces se rechaza la hipótesis.

Los resultados, a pesar de ser contrarios a lo que señala Castillo (6) que es muy difícil trabajar bajo esquemas asociativos por el interés individualista que tienen los empresarios y a lo que, Scott (9) en su informe de Competitividad agrícola y el desarrollo de cadenas y clusteres de valor en el Perú, señala que entre las limitaciones sociales del desarrollo de cadenas de valor y las empresas, una se refiere a las relaciones entre empresas y la falta de confianza entre ellas, por diferentes motivos como la cultura individualista y los intentos de imponer colaboración desde arriba; si corroboran lo que muestra Robbins (12) en los Valores Culturales de Hofstede por Naciones, ubicando al Perú con un índice de individualidad de 16 (sobre 100) y calificación 45, que indica su tendencia cultural nacional hacia el colectivismo y en el Valle del Mantaro, el rasgo de individualismo solo se presenta en un 16,47 $\%$ de los productores.

En relación con la importancia de la capacitación en temas productivos, asociatividad, trabajo en equipo, se tiene el aporte de Amartya Sen que señala: "Cuando se aplica el enfoque sobre la capacidad a la ventaja de una persona, lo que interesa es evaluarla en términos de su habilidad real para lograr funcionamientos valiosos como parte de la vida" (13). Esta cita grafica la aplicación del desarrollo de capacidades a nivel de los individuos. Es relevante mencionarla ya que en la medida en que estas capacidades calen en los productores pobres y pasen de ser meros conocimientos a convertirse en habilidades, se abrirá la posibilidad de que estos, a partir de la empresarialidad - en tanto que capacidad- puedan superar sostenidamente 
la pobreza. Por tanto la capacitación y asistencia técnica deben enfocarse en el logro de habilidades o capacidades, no solo en el traslado de conocimientos y los productores darán mayor valor a estas actividades de la cadena productiva.

En la dimensión desconfianza, sí se suma respuestas muy positivas y positivas como elementos contrarios al del rasgo de desconfianza, se tiene que el 51,6 $\%$ de productores manifiestan buena comunicación en la cadena productiva; un $40,2 \%$ de productores aportaron entre el $50 \%$ y $100 \%$ de los costos de producción; un $56 \%$ están dispuestos a aportar entre el $50 \%$ y $100 \%$ de los costos de producción en una nueva cadena productiva; un $60 \%$ confían en la empresa articuladora y un 60 $\%$ confían en sus colegas productores, se deduce que el 53,6 \% de los productores agrícolas del Valle del Mantaro no tienen el rasgo de desconfianza; y para el 46,4 $\%$ de productores del Valle del Mantaro si tienen el rasgo de desconfianza. Este valor si bien es menor, es importante porque podría influir negativamente en el trabajo asociativo de las cadenas productivas de agroexportación en el Valle del Mantaro, Junín, entonces se acepta la hipótesis 2, que la desconfianza para un 46,4 \% de productores del Valle del Mantaro, influye negativamente en el trabajo asociativo de una cadena productiva de agroexportación.

Esto corrobora lo que plantean Antezana, Bernet y otros (14), sobre la construcción de confianza entre los diferentes actores, que servirán para confirmar que la confianza es un elemento fundamental en el éxito de las cadenas productivas. La confianza permite que los actores se comuniquen más eficientemente, desarrollen una visión compartida e implementen estratégicamente actividades que puedan poner esa visión en práctica. Mientras más alto sea el nivel de confianza, mejores serán los resultados esperados en los procesos colaborativos. La condición de confianza en una empresa agroexportadora se daría siempre que ofrezcan garantía, responsabilidad, mercado seguro y propuestas claras, confirmando lo señalado por Laurence
Cornu (15) sobre la confianza que la conceptúa como la seguridad o esperanza firme que alguien tiene de otro individuo o de algo.

Se concluye, entonces, que solo el 16,47 $\%$ de productores del Valle del Mantaro tienen el rasgo de individualismo y este valor no influye negativamente en el trabajo asociativo de las cadenas productivas de agroexportación en el Valle del Mantaro, Junín, y el $46,4 \%$ de productores del Valle del Mantaro, tienen el rasgo de desconfianza y este valor sí influye negativamente en el trabajo asociativo de una cadena productiva de agroexportación en el Valle del Mantaro. Por lo que en futuras cadenas productivas de agroexportación que se deseen formar, se deberán tener en cuenta que la mayoría de productores del Valle del Mantaro no tienen el rasgo de individualismo, que tienen una buena actitud hacia el trabajo asociativo y en equipo, pero que las propuestas técnicas, económicas y de comercialización estén claras y haya responsabilidad y seriedad en la empresa articuladora.

\section{REFERENCIAS BIBLIOGRÁFICAS}

1. Avendaño TE, Roeland D, Risco M.M, Martínez A.A, Quispe PR, Pérez C.L, et al. Conociendo la Cadena Productiva de Tuna y Cochinilla en Ayacucho. Ayacucho: Solid Perú; 2008. Disponible en: http://www.solidperu.com/upl/1/ default/doc/Conociendo la cadena de la tuna y cochinilla en Ayacucho.pdf

2. Benavides M. Programa de competitividad - Corporación Andina de Fomento, CAF. Asociatividad y Cluster. Lima: Ministerio de Trabajo y Promoción del Empleo/PROMPYME. Disponible en: http://www.uss.edu.pe/uss/eventos/ JovEmp/pdf/ClusterdeCalzado.pdf

3. PROEXPANSION. Documento de Trabajo: Estudio sobre Cluster y Asociatividad. Lima: Ministerio de Trabajo y Promoción del Empleo/ PROMPYME. Disponible en: http:// www.uss.edu.pe/uss/eventos/JovEmp/ 
pdf/ClusterdeCalzado.pdf

4. Boucher F. Una visión territorial de la agroindustria rural: Los sistemas agroalimentarios localizados. Lima: PRODAR/IICA-CIRAD; 2000.

5. Latinobarómetro [Internet]. Chile: Corporación Latinobarómetro; 2013 [Citado 14 de marzo de 2014]. Disponible en: http://www. latinobarometro.org/ano2001/ grpre2002.pdf

6. Castillo A. Asociatividad. Lima: Ministerio de Trabajo y Promoción del Empleo/PROMPYME. Disponible en: http://www.uss.edu.pe/uss/eventos/ JovEmp/pdf/ClusterdeCalzado.pdf

7. Agroesan [Internet]. Lima: Elliot Arteaga Reyes; 2013. [Citado 14 de marzo de 2014]. Disponible en: http://www.agroesan.edu.pe/ columnistas? |ayout $=$ edit\&id $=188$

8. Instituto Nacional de Estadística e Informática. IV Censo Nacional Agropecuario IV CENAGRO. Lima: INEI; 2012.

9. Scott G. Competitividad agrícola y el desarrollo de cadenas y clusteres de valor en el Perú. Lima: BID; 2011. Disponible en: http://idbdocs. $\mathrm{iadb}$.org/wsdocs/getdocument. aspx? docnum $=35839504$

10. Hernández S.R, Fernández C.C, Baptista L.P Metodología de la investigación. $5^{\mathrm{a}}$ ed. México: Mc-Graw Hill; 2010.

11. Instituto Nacional de Estadística e Informática. Censos Nacionales 2007; XI Población y Vivienda, Perú: INEI; 2007.

12. Robbins S, Judge T. Comportamiento Organizacional. $13^{\mathrm{a}}$ ed. México: Pearson Prentice Hall; 2009.

13. Sen A. Desarrollo y Libertad. Buenos Aires: Planeta; 2000.

14. Antezana I, Thomas B, López G. Enfoque Participativo en Cadenas Productivas (EPCP). Lima: Centro Internacional de la Papa; 2008.

15. Cornu L, Douailler S, Lambruschini $G$, Tassin E, Vermeren $P$, Frigerio $G$. La confianza en las relaciones pedagógicas. Buenos Aires: Santillana; 2002. 